\title{
Open book decompositions of fiber sums in contact topology
}

\author{
MIRKO KLUKAS
}

\begin{abstract}
We describe compatible open books for the fiber connected sum along binding components and along multisections of open books. As an application, the first description provides simple ways of constructing open books supporting all tight contact structures on $T^{3}$, recovering a result by Van Horn-Morris, as well as an open book supporting the result of a Lutz twist along a binding component of an open book, recovering a result by Ozbagci and Pamuk.
\end{abstract}

57M50, 57MXX

\section{Introduction}

According to a theorem of Alexander [1], every closed oriented 3-manifold admits a so-called open book decomposition. While it has been known for almost 40 years that open books carry a natural contact structure (see Thurston and Winkelnkemper [8]) thanks to the work of Giroux [4] it turns out that this is just one fragment of a much deeper correlation. He showed that contact structures in dimension 3 are of purely topological nature and established a one-to-one correspondence between isotopy classes of contact structures and open book decompositions up to positive stabilization. Ever since, it has been of interest to recover properties and constructions of contact structures in the language of open books.

In the present paper we approach how the construction of the fiber connected sum affects an underlying open book structure of the original manifold under certain assumptions. Fiber connected sums in the contact setting recently appeared in Wendl's work [10] on planar torsion, an obstruction for strong fillability generalizing overtwistedness and Giroux torsion. In essence, a contact manifold admits planar torsion if it can be written as the binding sum - the fiber connected sum along binding components of an open book - of at least two open books, one of which has planar pages.

The present paper splits into two parts, Section 3 and Section 4, which can be read independently. The main results are descriptions of compatible open books for 
(I) the fiber connected sum along binding components of open books (Theorem 3.4), (II) the fiber connected sum along multisections of open books (Theorem 4.5).

In Section 3A we present some applications of these constructions, eg the first description provides simple ways of constructing open books supporting all tight contact structures on $T^{3}$, recovering a result by Van Horn-Morris [9], as well as an open book supporting the result of a Lutz twist along a binding component of an open book, recovering a result by Ozbagci and Pamuk [6].

Acknowledgements The results presented in this paper are part of my thesis. I want to thank my advisor Hansjörg Geiges for introducing me to the world of contact topology and for initiating the first part of the present paper. I deeply thank John Etnyre for many inspiring conversations and, in particular, for prompting the second part of this paper. I also thank the referee for useful comments that helped improve the exposition.

The research for the second part of this paper took place during a research visit at the Georgia Institute of Technology under the supervision of John Etnyre, and I thank them for their hospitality. This research visit was additionally supported by the DAAD (German Academic Exchange Service). The author was supported by the DFG (German Research Foundation) as fellow of the graduate training program Global structures in geometry and analysis at the mathematics department of the University of Cologne, and by grant GE 1245/2-1 to H Geiges.

\section{Preliminaries}

\section{A Open books}

An open book decomposition of a 3-dimensional manifold $M$ is a pair $(B, \pi)$, where $B$ is a disjoint collection of embedded circles in $M$ called the binding of the open book, and $\pi: M \backslash B \rightarrow S^{1}$ is a (smooth, locally trivial) fibration such that each fiber $\pi^{-1}(\varphi), \varphi \in S^{1}$, corresponds to the interior of a compact hypersurface $\Sigma_{\varphi} \subset M$ with $\partial \Sigma_{\varphi}=B$. The hypersurfaces $\Sigma_{\varphi}, \varphi \in S^{1}$, are called the pages of the open book.

In some cases we are not interested in the exact position of the binding or the pages of an open book decomposition inside the ambient space. Therefore, given an open book decomposition $(B, \pi)$ of a 3 -manifold $M$, we could ask for the relevant data to remodel the ambient space $M$ and its underlying open books structure $(B, \pi)$, say up to diffeomorphism. This leads us to the following notion.

An abstract open books is a pair $(\Sigma, \phi)$, where $\Sigma$ is a compact surface with non-empty boundary $\partial \Sigma$, called the page and $\phi: \Sigma \rightarrow \Sigma$ is a diffeomorphism equal to the identity 
near $\partial \Sigma$, called the monodromy of the open book. Let $\Sigma(\phi)$ denote the mapping torus of $\phi$, that is, the quotient space obtained from $\Sigma \times[0,1]$ by identifying $(x, 1)$ with $(\phi(x), 0)$ for each $x \in \Sigma$. Then the pair $(\Sigma, \phi)$ determines a closed manifold

$$
M_{(\Sigma, \phi)}:=\Sigma(\phi) \cup_{\text {id }}\left(\partial \Sigma \times D^{2}\right),
$$

where we identify $\partial \Sigma(\phi)=\partial \Sigma \times S^{1}$ with $\partial\left(\partial \Sigma \times D^{2}\right)$ using the identity map. Let $B \subset M_{(\Sigma, \phi)}$ denote the embedded link $\partial \Sigma \times\{0\}$. Then we can define a fibration $\pi: M_{(\Sigma, \phi)} \backslash B \rightarrow S^{1}$ by

$$
\left.\begin{array}{l}
{[x, \varphi]} \\
{\left[\theta, r \mathrm{e}^{i \pi \varphi}\right]}
\end{array}\right\} \mapsto[\varphi]
$$

where we understand $M_{(\Sigma, \phi)} \backslash B$ as decomposed as in (1) and $[x, \varphi] \in \Sigma(\phi)$ or $\left[\theta, r \mathrm{e}^{i \pi \varphi}\right] \in \partial \Sigma \times D^{2} \subset \partial \Sigma \times \mathbb{C}$ respectively. Clearly $(B, \pi)$ defines an open book decomposition of $M_{(\Sigma, \phi)}$.

On the other hand, an open book decomposition $(B, \pi)$ of some 3-manifold $M$ defines an abstract open book as follows: identify a neighborhood of $B$ with $B \times D^{2}$ such that $B=B \times\{0\}$ and such that the fibration on this neighborhood is given by the angular coordinate, $\varphi$ say, on the $D^{2}$-factor. We can define a 1 -form $\alpha$ on the complement $M \backslash\left(B \times D^{2}\right)$ by pulling back $d \varphi$ under the fibration $\pi$, where this time we understand $\varphi$ as the coordinate on the target space of $\pi$. The vector field $\partial \varphi$ on $\partial\left(M \backslash\left(B \times D^{2}\right)\right)$ extends to a nowhere vanishing vector field $X$ which we normalize by demanding it to satisfy $\alpha(X)=1$. Let $\phi$ denote the time 1 map of the flow of $X$. Then the pair $(\Sigma, \phi)$, with $\Sigma=\overline{\pi^{-1}(0)}$, defines an abstract open book such that $M_{(\Sigma, \phi)}$ is diffeomorphic to $M$.

2A1 Examples Understand $S^{3}$ as the unit sphere in $\mathbb{C}^{2}$, ie as the subset of $\mathbb{C}^{2}$ given by

$$
S^{3}=\left\{\left(z_{1}, z_{2}\right) \in \mathbb{C}^{2}:\left|z_{1}\right|^{2}+\left|z_{2}\right|^{2}=1\right\} .
$$

We give three examples of open book decompositions of $S^{3}$ :

(1) Set $B=\left\{\left(z_{1}, z_{2}\right) \in S^{3}: z_{1}=0\right\}$. Note that $B$ is an unknotted circle in $S^{3}$. Consider the fibration

$$
\pi: S^{3} \backslash B \rightarrow S^{1} \subset \mathbb{C}, \quad\left(z_{1}, z_{2}\right) \mapsto z_{1} /\left|z_{1}\right| .
$$

In polar coordinates this map is given by $\left(r_{1} \mathrm{e}^{i \varphi_{1}}, r_{2} \mathrm{e}^{i \varphi_{2}}\right) \mapsto \varphi_{1}$. Observe that $(B, \pi)$ defines an open book decomposition of $S^{3}$ with pages diffeomorphic to $D^{2}$ and trivial monodromy. 
(2) Set $B_{+}=\left\{\left(z_{1}, z_{2}\right) \in S^{3}: z_{1} z_{2}=0\right\}$. Observe that $B_{+}$describes the positive Hopf link. Consider the fibration

$$
\pi_{+}: S^{3} \backslash B_{+} \rightarrow S^{1} \subset \mathbb{C}, \quad\left(z_{1}, z_{2}\right) \mapsto \frac{z_{1} z_{2}}{\left|z_{1} z_{2}\right|} .
$$

One can show that $\left(\pi_{+}, B_{+}\right)$defines an open book decomposition of $S^{3}$ with annular pages and monodromy given by a right-handed Dehn twist along the core of the annulus.

(3) Set $B_{-}=\left\{\left(z_{1}, z_{2}\right) \in S^{3}: z_{1} \bar{z}_{2}=0\right\}$. Observe that $B_{-}$describes the negative Hopf link. Consider the fibration

$$
\pi_{-}: S^{3} \backslash B_{-} \rightarrow S^{1} \subset \mathbb{C}, \quad\left(z_{1}, z_{2}\right) \mapsto \frac{z_{1} \bar{z}_{2}}{\left|z_{1} \bar{z}_{2}\right|} .
$$

One can show that $\left(\pi_{-}, B_{-}\right)$defines an open book decomposition of $S^{3}$ with annular pages and monodromy given by a left-handed Dehn twist along the core of the annulus.

\section{B Stabilization of open books}

Let $\Sigma$ be a compact surface with non-empty boundary and $\phi: \Sigma \rightarrow \Sigma$ be a diffeomorphism equal to the identity near $\partial \Sigma$. Suppose further we are given a properly embedded arc $a \subset \Sigma$. The positive (negative) stabilization of the abstract open book $(\Sigma, \phi)$ is the abstract open book obtained by adding a 1-handle to the original page $\Sigma$ along the endpoints of $a$, and changing the monodromy by composing it with a right(left-) handed Dehn twist along the simple closed curve obtained by the union of $a$ and the core of the 1-handle. The open books described in parts (2) and (3) of the preceding example are instances of a positive and negative stabilization respectively of the open book described in the first part.

A positive and negative stabilization respectively can be understood as a suitable connected sum with the open book described in part (2) or part (3) of the preceding example. This viewpoint will draw more attention once we introduced the interplay of open books and contact structures; see Section 2C below. To be more precise, a positive stabilization of an open book does not change the underlying contact structure, whereas a negative stabilization turns it into an overtwisted one.

\section{C Compatibility}

A positive contact structure $\xi=\operatorname{ker} \alpha$ and an open book decomposition $(B, \pi)$ of $M$ are said to be compatible with each other, if the 2 -form $d \alpha$ induces a symplectic form 
on each page, defining its positive orientation, and the 1 -form $\alpha$ induces a positive contact form on $B$.

In dimension equal to 3 it can be shown that any two contact structures supported by the same open book decomposition are in fact contact isotopic; see [2]. The open books described in parts (1) and (2) of Section 2A1 above support the standard contact structure $\xi_{\text {st }}$ on $S^{3}$, whereas part (3) supports the overtwisted contact structure $\xi_{1}$ which is obtained by a Lutz twist along a transverse unknot $U \subset\left(S^{3}, \xi_{\text {st }}\right)$ with self-linking number -1 .

\section{D The fiber connected sum}

Let us recall the definition of the fiber connected sum in dimension 3. For a more precise and general description of the fiber connected sum along codimension 2 contact submanifolds, see [3, Section 7.4].

Let $K \subset(M, \xi)$ be a positive transverse knot sitting in a contact 3 -manifold $(M, \xi)$. We may identify a neighborhood of $K$ with an $\varepsilon$-neighborhood $N_{\varepsilon} \subset S^{1} \times \mathbb{R}^{2}$, where $K=S^{1} \times\{0\}$. Then, with $S^{1}$-coordinate $\theta$, polar coordinates $(r, \varphi)$ on $\mathbb{R}^{2}$, and for a suitable $\varepsilon>0$, the contact structure

$$
d \theta+r^{2} d \varphi=0
$$

provides a model for the above neighborhood of $K$. Let $M_{K}=M \backslash N_{\delta}$ denote the complement of a $\delta$-neighborhood $N_{\delta} \subset N_{\varepsilon}$, with $0<\delta<\varepsilon$, where we change the contact structure as follows. Replace the contact structure $\xi$ over $N_{\varepsilon} \backslash N_{\delta}$ by the kernel of the contact 1 -form $d \theta+f(r) d \varphi$, where $f:[\delta, \varepsilon] \rightarrow \mathbb{R}$ is a function that equals $r^{2}$ near $\varepsilon$, satisfies $f^{\prime}>0, f^{\prime}(\delta)=1$ and $f(\delta)=0$. We will refer to $M_{K}$ as obtained by blowing up $K$. The inverse operation of blowing up will be referred to as collapsing.

Suppose now we are given a pair of positive transverse knots $K_{0}, K_{1} \subset(M$, $\xi)$ which are endowed with a framing. Let $M_{K_{0}, K_{1}}$ denote the result of blowing up each of the knots $K_{0}$ and $K_{1}$. Each of the boundary tori associated to $K_{0}$ and $K_{1}$ respectively admits a natural identification with $\mathbb{R}^{2} / \mathbb{Z}^{2}$ by sending the meridian to the $x$-axis and the framing direction to the $y$-axis. The fiber connected sum $\#_{K_{0}, K_{1}}(M, \xi)$ is the closed oriented manifold given as the quotient space

$$
\underset{K_{0}, K_{1}}{\#}(M, \xi):=\left(M_{K_{0}, K_{1}}\right) / \sim,
$$

where we identify the boundary tori with respect to the gluing map sending $(x, y)$ to $(-x, y)$. Sometimes we identify the framing direction with the $y$-axis with respect to previously chosen parametrizations of $K_{0}$ and $K_{1}$. This has no effect on the final 
contact manifold. However it is necessary to avoid ambiguities if we want to keep track of the change of additional structures on $M$ (eg open book decompositions).

\section{An open book supporting the binding sum}

In the previous section we introduced the fiber connected sum along a pair of positively transverse knots in a contact manifold. In the present section we proceed by considering two special cases, the fiber connected sum along binding components of open books and the fiber connected sum along sections of open books. Throughout the whole section let $(M, \xi)$ be a closed, not necessarily connected, contact 3-manifold supported by an open book $(\Sigma, \phi)$. Let $B \subset M$ denote the embedded binding of the open book.

Suppose we have chosen the transverse knots $K_{0}$ and $K_{1}$ to be components of the binding of the open book decomposition $(\Sigma, \phi)$. Since the pages induce a natural framing for $K_{0}$ and $K_{1}$ respectively we can think of it as the zero-framing and hence can measure all other trivializations relative to it. Note that performing the fiber connected sum with framings $m_{1}, m_{2} \in \mathbb{Z}$ equals the result of performing the fiber connected sum with framings $\widetilde{m}_{1}=m_{1}+m_{2}$ and $\widetilde{m}_{2}=0$. So in the following we just fix one framing assuming the other one to be zero. The result of performing the fiber connected sum along two binding components $K_{0}$ and $K_{0}$ with framing $m \in \mathbb{Z}$ will be referred to as binding sum along $K_{0}$ and $K_{1}$ and will be denoted by

$$
\bigoplus_{m}(\Sigma, \phi) \text {. }
$$

Now suppose $K_{0}$ and $K_{1}$ are positive transverse knots intersecting every page transversely and exactly once. We will refer to such a knot as a section of the open book, since it induces a section of the fibration $\pi: M \backslash B \rightarrow S^{1}$ associated to the open book $(\Sigma, \phi)$. Furthermore we can identify $K_{i}$ with $S^{1}$ via the projection $\left.\pi\right|_{K_{i}}$ for $i=0,1$. By nature of the sections we can embed the normal bundle $N_{i}, i=0,1$, such that each fiber $\left(N_{i}\right)_{\varphi}, \varphi \in S^{1}$, corresponds to a disc neighborhood $D_{i} \subset \Sigma_{\varphi}$ of the intersection point $\left\{p_{i}\right\}=K_{i} \cap \Sigma_{\varphi}$. In the following we will see that fiber connected sums of this kind are nicely adapted to the underlying open book decomposition, in the sense that the result again admits an open book.

The new page is obtained by replacing $D_{0} \cup D_{1} \subset \Sigma_{\varphi}$ with $D^{1} \times S^{1}$. However, the change of monodromy is less obvious. To see how the monodromy changes, consider a vector field transverse to the fibers in $M$ with $K_{0}$ and $K_{1}$ as closed orbits such that the return map $h$ on a fiber $\Sigma$ fixes a disc neighborhood $D_{i}$ of each $\Sigma \cap K_{i}$ and such that closed orbits close to $K_{0}$ and $K_{1}$ represent the trivializations of the sections. The new monodromy is equal to $h$ on $\Sigma \backslash\left(D_{0} \cup D_{1}\right)$ and the identity on $D^{1} \times S^{1}$. 
For our purposes it will be sufficient just to consider trivial sections, that is, sections corresponding to a single fixed point $p \in \Sigma$ of the monodromy of a given abstract open book $(\Sigma, \phi)$. In this case we obtain natural trivializations of the normal bundles given by a parallel copy of the knot corresponding to a nearby point. Furthermore we can assume the given monodromy $\phi$ to be the identity on $D_{0} \cup D_{1}$. So by the observations above, the new monodromy will be given by $\phi$ on $\Sigma \backslash\left(D_{0} \cup D_{1}\right)$ and the identity on $D^{1} \times S^{1}$.

Lemma 3.1 Let $K$ be a section of an open book $(B, \pi)$ supporting a contact 3manifold $\left(M, \xi_{0}=\operatorname{ker} \alpha_{0}\right)$ and let $(\theta, r, \varphi)$ denote coordinates on $S^{1} \times \mathbb{R}^{2}$ with $(r, \varphi)$ being polar coordinates on $\mathbb{R}^{2}$. Then there is another compatible contact structure $\xi_{1}=\operatorname{ker} \alpha_{1}$ and a neighborhood $N$ of $K$ together with an embedding $N \hookrightarrow S^{1} \times \mathbb{R}^{2}$ such that

- $K$ maps to $S^{1} \times\{0\}$,

- $\pi$ corresponds to the projection on the $S^{1}$-factor, and

- the contact form $d \theta+r^{2} d \varphi$ pulls back to $\alpha_{1}$.

In particular, each intersection point of $K$ and any page $\Sigma_{\varphi}, \varphi \in S^{1}$, corresponds to an elliptic singularity of the characteristic foliation $\left(\Sigma_{\varphi}\right)_{\xi_{1}}$. The analogous statement holds for multisections of open books as defined in Section 4.

Proof Fix a page $\Sigma=\Sigma_{\varphi}$ of $(B, \pi)$ and let $N(B)$ denote a neighborhood of the binding disjoint from $K$. Furthermore let $p=\Sigma \cap K$ denote the transverse intersection of $K$ and $\Sigma$. Identify the complement $M \backslash N(B)$ of $N(B)$ with the mapping torus $\Sigma(\phi)$ of a suitable monodromy map $\phi: \Sigma \rightarrow \Sigma$, ie we have

$$
M \backslash N(B) \cong \Sigma \times[0,1] / \sim,
$$

where we identify $(x, 1)$ with $(\phi(x), 1)$ for all $x \in \Sigma$. Note that we may assume that we have chosen $\phi$ in such a way that, with respect to the above identification, $\{p\} \times[0,1]$ descends to $K$. Moreover we can assume $\phi$ to fix a little neighborhood of $p$. Hence it remains to show that there is a contact structure on $\Sigma(\phi)$ satisfying the desired property for this particular case.

Following the construction of Thurston and Winkelnkemper [8] all we have to do is choose an exact volume form $d \beta$ on $\Sigma$ such that the Liouville vector field $Y$, associated to the primitive $\beta$, points outwards along $\partial \Sigma$ and has an elliptic singularity at $p$.

Lemma 3.2 The contact manifold resulting from the (contact) fiber connected sum along two sections of an open book is compatible with the corresponding open book. The analogous statement holds for multisections of open books as defined in Section 4. 
Proof Let $K_{0}$ and $K_{1}$ denote two framed sections in a (not necessarily connected) contact manifold $(M, \xi)$ supported by an open book $(B, \pi)$. Let $N_{0}$ and $N_{1}$ denote small disjoint neighborhoods of $K_{0}$ and $K_{1}$ each of which we identify with a copy of $S^{1} \times D_{\varepsilon}^{2}$. According to Lemma 3.1 we can assume that (after an isotopy) $\xi$ is given by the kernel of a 1 -form $\alpha$ such that over each copy of $S^{1} \times D_{\varepsilon}^{2}$ the 1 -form $\alpha$ is given by $d \theta+r^{2} d \varphi$ and $\pi$ is given by the projection on the $S^{1}$-factor.

Let $(\theta, r, \varphi)$ denote coordinates on $S^{1} \times[-\varepsilon, \varepsilon] \times S^{1}$ and let $\xi^{\prime}$ be the kernel of the contact form $d \theta+f(r) d \varphi$, where $f:(-\varepsilon, \varepsilon) \rightarrow \mathbb{R}$ is a function satisfying the following properties:

- $f(r)=\mp r^{2}$ near $\pm \varepsilon$

- $f^{\prime}>0$,

- $f(0)=0$.

The fiber connected sum along $K_{0}$ and $K_{1}$ is given as follows. Consider the complement $M \backslash\left(N_{0} \times N_{1}\right)$ of the neighborhoods $N_{0}$ and $N_{1}$ of our given sections. Each of the boundary tori $T_{0}, T_{1}$ associated to $K_{0}$ and $K_{1}$ respectively admits a natural identification with $\mathbb{R}^{2} / \mathbb{Z}^{2}$ by sending the meridian to the $x$-axis and the framing direction to the $y$-axis. The fiber connected sum $\#_{K_{0}, K_{1}}(M, \xi)$ is given as the quotient space

$$
\left(M \backslash\left(N_{0} \times N_{1}\right), \xi\right) \cup \Psi\left(S^{1} \times[-\varepsilon, \varepsilon] \times S^{1}, \xi^{\prime}\right),
$$

where we glue with respect to the map

$$
\Psi(x, y):= \begin{cases}(y,-\varepsilon, x) & \text { for }(x, y) \in T_{0}, \\ (y, \varepsilon,-x) & \text { for }(x, y) \in T_{1} .\end{cases}
$$

Over $M \backslash\left(N_{0} \times N_{1}\right)$ the contact structure and the open book already are compatible. Over the remaining part $S^{1} \times[-\varepsilon, \varepsilon] \times S^{1}$ this the case as well since $f^{\prime} d r \wedge d \varphi$ descends to a symplectic form on the pieces $\{\theta\} \times[-\varepsilon, \varepsilon] \times S^{1}, \theta \in S^{1}$.

Given a simple closed curve $\alpha$ on some surface $\Sigma$ we denote by $\tau_{\alpha}$ and $\left(\tau_{\alpha}\right)^{-1}$ respectively the right- and left-handed Dehn twist along $\alpha$. When we deal with the concatenation of Dehn twists we sometimes omit the concatenation symbol $\circ$ to simplify the notation. In this fashion it makes sense to consider $n^{\text {th }}$-powers $\left(\tau_{\alpha}\right)^{n}$ of Dehn twists, where the zero power $\left(\tau_{\alpha}\right)^{0}$ is defined to be the identity map.

Let $K \subset \partial \Sigma$ denote a boundary component of the page $\Sigma$ provided with some framing $m \in \mathbb{Z}$ and let $K^{\prime} \subset \Sigma$ denote the transverse knot indicated by the black dot in Figure 1, in which we understand $K^{\prime}$ as zero-framed. The framings are understood as measured with respect to their respective natural zero-framings as explained above. 
Definition 3.3 We will refer to a framed binding component $(K, m)$ as admitting a navel if the monodromy near the boundary component is given by $\tau_{\alpha} \tau_{\beta}^{-1} \tau_{\gamma}^{m-1}$, where the curves $\alpha, \beta, \gamma \subset \Sigma$ are given as in Figure 1. The transverse knot $K^{\prime}=\left(K^{\prime}, 0\right)$ indicated by the black dot in Figure 1 will be referred to as the core of the navel corresponding to $(K, m)$. Observe that we can change every boundary component into a navel, since the monodromy $\tau_{\alpha} \tau_{\beta}^{-1} \tau_{\gamma}^{m-1}$ is isotopic to the identity.

In the following theorem, we express the binding sum as the fiber sum along the core of its corresponding navel.

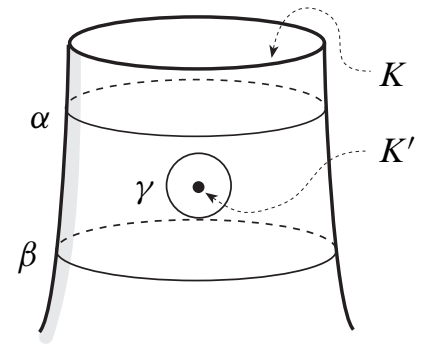

Figure 1: Binding component admitting a navel.

Theorem 3.4 Let $K \subset \partial \Sigma$ be a binding component provided with a framing $m \in \mathbb{Z}$. Then the framed knot $(K, m)$ is transversely isotopic to the corresponding core $\left(K^{\prime}, 0\right)$ of its navel. In consequence the result of performing the binding sum with framing $m \in \mathbb{Z}$ along two binding components $K_{0}, K_{1} \subset \Sigma$ corresponds to the fiber sum along the cores $K_{0}^{\prime}, K_{1}^{\prime}$ of their corresponding navels; see also Figure 2.

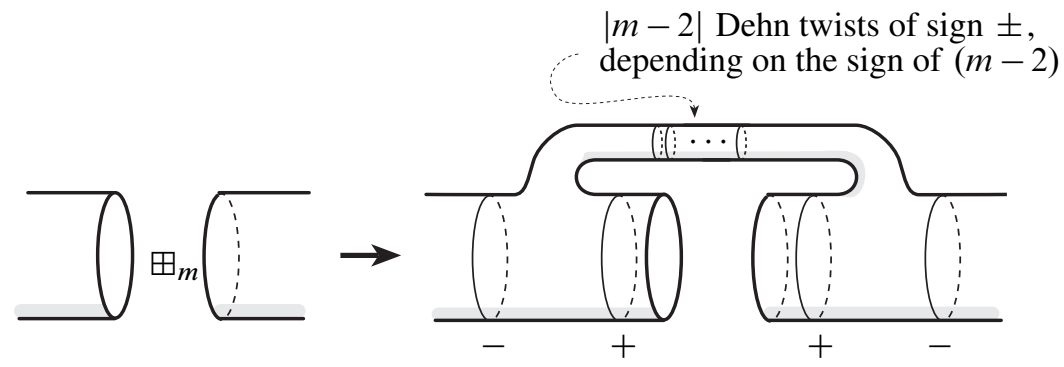

Figure 2: An open book supporting the binding sum.

Proof Identify a neighborhood of the binding component $K \subset \partial \Sigma$ with an $\varepsilon-$ neighborhood $N_{\varepsilon} \subset S^{1} \times \mathbb{R}^{2}$, where $K=S^{1} \times\{0\}$. Then, with $S^{1}$-coordinate 
$\theta$, polar coordinates $(r, \varphi)$ on $\mathbb{R}^{2}$, and for a suitable $\varepsilon>0$, the contact structure

$$
d \theta+r^{2} d \varphi=0
$$

provides a model for the above neighborhood of $K$. Moreover we can assume that over this neighborhood the pages are given by the preimages of the projection on the angular coordinate $\varphi$, ie the closure of every page can be described as $A_{\varphi}=S^{1} \times[0, \varepsilon] \times\{\varphi\}$ for some appropriate $\varphi \in S^{1}$.

We will now apply the first part $\tau_{\alpha} \tau_{\beta}^{-1}$ of the monodromy of the navel (the twists $\tau_{\gamma}^{m-1}$ just take care of the framings, but we will come to that later). Let $S^{1} \times[\delta, \varepsilon] \times S^{1}$ denote the complement of a $\delta$-neighborhood $N_{\delta}$ of $K$ in $N_{\varepsilon}$ for some small $0<\delta<\varepsilon$. Consider the map $\phi: S^{1} \times[\delta, \varepsilon] \rightarrow S^{1} \times[\delta, \varepsilon]$ defined by

$$
\phi(\theta, r):=(\theta+h(r), r),
$$

where $h:[\delta, \varepsilon] \rightarrow[0,1]$ is the function satisfying the following properties:

- $h(r)=0$ for $r$ near $\delta$ and near $\varepsilon$.

- $h(r)=1$ on an interval containing $(\varepsilon+\delta) / 2$.

- $h^{\prime}(r) \geq 0$ for $r<(\varepsilon+\delta) / 2$.

- $h^{\prime}(r) \leq 0$ for $r>(\varepsilon+\delta) / 2$.

Note that with respect to the identification $S^{1} \equiv \mathbb{R} / \mathbb{Z}$ the map $\phi$ is indeed well defined and observe that $\phi$ equals $\tau_{\alpha} \tau_{\beta}^{-1}$ and is isotopic to the identity. Consider the corresponding mapping torus $A(\phi)$, that is

$$
A(\phi)=\left(S^{1} \times[\delta, \varepsilon] \times[0,1]\right) / \sim_{\phi},
$$

where we identify $(\theta+h(r), r, 1)$ with $(\theta+h(r), r, 0)$ for each $(\theta, r) \in S^{1} \times[\delta, \varepsilon]$. Following the construction of Thurston and Winkelnkemper [8] we can endow $A(\phi)$ with the contact structure $\xi^{\prime}$ given by the kernel of the contact 1 -form

$$
\left((1-\varphi) d \theta+\varphi \phi^{*} d \theta\right)+r^{2} d \varphi
$$

(actually this defines a contact structure on $S^{1} \times[\delta, \varepsilon] \times[0,1]$ that descends to a contact structure on $A(\phi))$. Observe that, since $\phi$ is isotopic to the identity, $\left(A(\phi), \xi^{\prime}\right)$ and $\left(S^{1} \times[\delta, \varepsilon] \times S^{1}, \xi\right)$ are contactomorphic under a contactomorphism keeping little neighborhoods of the boundary fixed. The space $A(\phi)$ is foliated by tori $T_{r}$ of the form

$$
T_{r}=\left(S^{1} \times\{r\} \times[0,1]\right) / \sim_{\phi},
$$


where we identify $(\theta, r, 1)$ with $(\theta+h(r), r, 0)$ for each $\theta \in S^{1}$. We can also understand these tori as the quotient of $\mathbb{R}^{2}$ and the lattice spanned by $(1,0)$ and $(h(r), 1)$ (in the same manner as we understand $S^{1} \times S^{1}$ as $\left.\mathbb{R}^{2} / \mathbb{Z}^{2}\right)$. The characteristic foliation $\left(T_{r}\right)_{\xi}$ of each torus is given by linear curves of slope $s\left(T_{r}\right)=-1 / r^{2}$, where we measure the slope with respect to the identification as above. Hence any closed curve $c$ on $T_{r}$ describes a transverse knot as long as the slope of $\dot{c}$ does not equal $-1 / r^{2}$. Now let $K^{+}$be the positive, transverse push-off of $K$, ie $K^{+}$is a linear curve on $T_{\delta}$ of slope +1 .

We are now going to define an isotopy $K_{S}$ of transverse knots connecting $K^{+}$with the core of the navel $K^{\prime}$. For $s \in[\delta,(\varepsilon+\delta) / 2]$ let $c_{S}:[0,1] \rightarrow[0,1] \times[0,1]$ denote the family of embedded curves with the following properties:

- $c_{s}(0)=(h(s), 0)$.

- $c_{S}(1)=(1,1)$.

- $\dot{c}_{S} \geq 0$.

- $\quad \dot{c}_{S}(0)=\dot{c}_{s}(1)= \pm \infty$.

Observe that each of the curves $c_{S}$ gives rise to a closed curve $K_{S}$ on $T_{S}$; see Equation (2) above. In particular these knots are transverse, since we have $\dot{c}_{s} \geq$ $0>s\left(T_{s}\right)$. Furthermore we have $K_{\delta}=K^{+}$and $K_{(\varepsilon+\delta) / 2}=K^{\prime}$.

Let us see what happens to the framing of the knots. Assume that the initial framing of $K$ was $m$. Observe that the framing of the transverse push-off $K^{+}$with respect to $T_{1}$ is given by $m-1$. The isotopy of knots $K_{s}^{+}$does not change the framing at all. Hence at this point we constructed a transverse isotopy connecting $(K, m)$ and $\left(K^{\prime}, m-1\right)$. Since the slope of $K^{\prime}$ equals $\infty$ we can apply the twists $\tau_{\gamma}^{m-1}$ around $K^{\prime}$ such that we end up with a zero-framed knot $K^{\prime}$ and we are done.

Example Consider two copies of the open book $\left(D^{2}\right.$, id) supporting $S^{3}$ with the standard contact structure $\xi_{\text {st }}$. It is easy to see that the result of the fiber connected sum along the only binding components yields $S^{1} \times S^{2}$ with its standard contact structure. Using the description of a compatible open book for the binding sum in Theorem 3.4 we obtain the standard open book description of $S^{1} \times S^{2}$ given by an annulus with trivial monodromy.

\section{A Applications}

We conclude the first part of the present paper with a few applications of the open book description given in Theorem 3.4. 
3A1 Tight contact structures on $\boldsymbol{T}^{\mathbf{3}}$ Let $\left(\theta_{1}, \theta_{2}, \varphi\right)$ denote coordinates on the 3 dimensional torus $T^{3}$ and consider the tight contact structure $\xi_{n}$ given by the kernel of the contact form $\cos (n \varphi) d \theta_{1}+\sin (n \varphi) d \theta_{2}$. The contact structures $\xi_{n}$ provide a complete list of tight contact structures on $T^{3}$ [5] and can also be described in the following way. Take $2 n$ copies of the open book $\left(S^{1} \times[0,1]\right.$, id), which is an open book compatible with the standard contact structure on $S^{1} \times S^{2}$, and then perform the $2 n$-fold binding sum in the obvious way. Now using Theorem 3.4 we are able to translate the above construction of $\left(T^{3}, \xi_{n}\right)$ into compatible open books. These open books for $\left(T^{3}, \xi_{n}\right)$ were first computed by Van Horn-Morris [9] using different methods than the ones presented here.

3A2 Full Lutz twist along binding component Consecutively performing the binding sum with two copies of the open book $\left(S^{1} \times[0,1]\right.$, id) has the effect of a full Lutz twist along the binding component. Again using Theorem 3.4 we are able to compute a compatible open book. One can show that this open book is stably equivalent to the compatible open book constructed by Ozbagci and Pamuk [6]. Obviously we can compute the effect of a regular Lutz twist in the same fashion.

3A3 Recovering Giroux torsion Let $(M, \xi)$ be a contact 3-manifold with nonzero Giroux torsion, ie if we choose $\left(\theta_{1}, \theta_{2}\right)$ to be coordinates on $T^{2}$ there exists an embedding of the contact manifold

$$
\left(T^{2} \times[0,2 \pi], \xi_{2 \pi}=\operatorname{ker}\left(\cos (t) d \theta_{1}+\sin (t) d \theta_{2}\right)\right)
$$

into $(M, \xi)$. So far, there was no way to recover Giroux torsion in the language of open books. We approach this question by computing a certain compatible open book for $(M, \xi)$.

Consider the complement $(M, \xi) \backslash\left(T^{2} \times[0,2 \pi], \xi_{1}\right)$ of the Giroux domain in $(M, \xi)$. The boundary of $(M, \xi) \backslash\left(T^{2} \times[0,2 \pi], \xi_{1}\right)$ consists of two pre-Lagrangian tori which are foliated by an $S^{1}$-family of closed curves. Collapsing these tori, in the sense of Section 2D, gives rise to a new closed contact manifold $\left(M^{\prime}, \xi^{\prime}\right)$ with two distinguished transverse knots $K_{0}$ and $K_{1}$. In this particular case we decorate these knots with the framing corresponding to the $\theta_{1}$ coordinate. Let $\left(\Sigma^{\prime}, \phi^{\prime}\right)$ denote a compatible open book decomposition of $\left(M^{\prime}, \xi^{\prime}\right)$ such that $K_{0}$ and $K_{1}$ are part of the binding $\partial \Sigma^{\prime}$. Let $m, n \in \mathbb{Z}$ be the above framings (induced by $\theta_{1}$ ) expressed with respect to the page-framing induced by the open book $\left(\Sigma^{\prime}, \phi^{\prime}\right)$.

Observe that if we take two copies of the open book $\left(S^{1} \times[0,1]\right.$, id), perform the binding sum along $S^{1} \times\{0\}$ in each copy of $\left(S^{1} \times[0,1]\right.$, id) and blow up the two remaining components corresponding to $S^{1} \times\{1\}$ in each copy we end up with $\left(T^{2} \times[0,2 \pi], \xi_{2 \pi}\right)$. 
Hence performing the 2 -fold binding sum of $\left(\Sigma^{\prime}, \phi^{\prime}\right)$ with $\left(S^{1} \times[0,1]\right.$, id $) \boxplus\left(S^{1} \times\right.$ $[0,1]$,id) along $K_{0}$ and the first copy of $S^{1} \times\{1\}$ and along $K_{1}$ and the second copy of $S^{1} \times\{1\}$ actually gives us a description of $(M, \xi)$ which, using Theorem 3.4, may be translated into an open book.

3A4 Surface bundles with invariant dividing set Let $\Sigma$ be a closed oriented surface containing a collection $\Gamma \subset \Sigma$ of oriented, mutually disjoint, embedded circles. Suppose there is a choice of orientations on the regions $\Sigma \backslash \Gamma$ which is coherent with the orientation of $\Gamma$. Let $\Sigma^{+}$and $\Sigma^{-}$denote the collections of positive and negative oriented regions of $\Sigma \backslash \Gamma$. We will refer to $(\Sigma, \Gamma)$ as an abstract convex surface. Let $\phi: \Sigma \rightarrow \Sigma$ denote a diffeomorphism that restricts to the identity in a neighborhood $N(\Gamma)$ of the abstract dividing set $\Gamma$. Write $\pi$ for the projection from the mapping torus $\Sigma(\phi)$ to the circle. Note that there is a natural contact structure $\xi_{\Gamma}$, such that for each $\theta \in S^{1}$ the fiber $\pi^{-1}(\theta) \cong \Sigma$ is a convex surface with dividing set $\Gamma$.

Write $\phi^{ \pm}$for the restrictions of $\phi$ to $\Sigma^{ \pm}$. Then we may identify the contact manifold $\left(\Sigma(\phi), \xi_{\Gamma}\right)$ with the fiber sum of $\left(\Sigma^{+}, \phi^{+}\right)$and $\left(\Sigma^{-}, \phi^{-}\right)$(where the latter objects are understood as open books), ie

$$
\left(\Sigma(\phi), \xi_{\Gamma}\right)=\left(\Sigma^{+}, \phi^{+}\right) \boxplus\left(\Sigma^{-}, \phi^{-}\right) .
$$

With the help of Theorem 3.4 this identification may be translated into an open book.

\section{Fiber connected sum along multisections}

Suppose we are given a 3-manifold $M$ admitting an open book decomposition $(\Sigma, \phi)$. An $n$-fold section of the open book is a knot $K \subset M$ that intersects every page transversely and exactly $n$ times, where the positive integer $n$ is called the index of $K$. Note that any $n$-fold section gives rise to a generalized braid in $\Sigma \times[0,1]$ whose induced permutation is cyclic, and vice versa, provided that the monodromy fixes the braid's endpoints. We will use the notions multisections and generalized braids almost interchangeably.

In the present section we try to approach the following question. Assume we are given two framed knots $K_{0}$ and $K_{1}$ embedded in 3-dimensional contact manifolds $\left(M_{0}, \xi_{0}\right)$ and $\left(M_{1}, \xi_{1}\right)$ respectively. Suppose further that, for $i=0,1$, the contact structure $\xi_{i}$ admits a compatible open book decomposition $\pi_{i}: M_{i} \backslash B_{i} \rightarrow S^{1}$ such that the knot $K_{i}$ provides an $n$-fold section of the open book for some mutual previously fixed positive integer $n \in \mathbb{N}$. For $i=0,1$ fix an identification of $K_{i}$ with the unit circle $S^{1} \subset \mathbb{C}$ such that the map $\left.\pi_{i}\right|_{K_{i}}: K_{i} \rightarrow S^{1}$ is given by $z \mapsto z^{n}$. Note that this identification 
is unique up to multiplication with $\mathrm{e}^{2 \pi i k / n}$ for some $k=1, \ldots, n-1$. Now perform the fiber connected sum along $K_{0}$ and $K_{1}$ with respect to the parametrizations above. Recall the discussion of the fiber sum along ordinary sections at the beginning of Section 3 and note that the open book decompositions $\left(\pi_{i}, B_{i}\right)$ descend to an open book decomposition

$$
\pi:\left(M_{0} \#_{K_{0}, K_{1}} M_{1}\right) \backslash\left(B_{0} \cup B_{1}\right) \rightarrow S^{1}
$$

on the fiber sum $M_{0} \#_{K_{0}, K_{1}} M_{1}$. As in the first part of the paper, we ask for an abstract description of the open book defined above in terms of the abstract descriptions of $\left(\pi_{i}, B_{i}\right)$. Observe that the page will be the $n$-fold connected sum of the two original pages. However it is not clear what the monodromy looks like. This question will be settled in the following subsections.

We assume that the reader is familiar with the basic notions of braid theory. For a brief introduction we point the reader to [7].

\section{A Standard braids and their induced sections}

Consider the unit disc $D^{2}$ together with a family of $n$ marked points $p_{1}<\cdots<p_{n}$ lying on the $x$-axis. Given an open book $(\Sigma, \phi)$ we fix an embedding of $D^{2}$ into a small neighborhood of the boundary $\partial \Sigma \subset \Sigma$ and denote by $j:\left(D^{2} \times[0,1]\right) \hookrightarrow(\Sigma \times[0,1])$ its obvious extension. We define the positive and the negative standard braid of index $n$ in $\Sigma \times[0,1]$ as

$$
j\left(S_{n}^{ \pm}\right) \subset \Sigma \times[0,1],
$$

where $S_{n}^{ \pm} \subset D^{2} \times[0,1]$ denote the braids given in Figure 3. For the sake of convenience we will continue to denote the standard braids as well as their induced sections by $S_{n}^{ \pm}$. Note that $S_{n}^{ \pm}$admits a natural framing induced by the $x$-direction. Furthermore we fix a parametrization of the $n$-fold section $S_{n}^{ \pm} \subset(\Sigma, \phi)$ over $S^{1}$ by sending $p_{1} \in S_{n}^{ \pm}$ to $1 \in S^{1} \subset \mathbb{C}$. When performing fiber sums along $S_{n}^{ \pm}$we always - unless stated otherwise - perform them with respect to these choices.

\section{B Monodromy corresponding to standard braids}

In the present section we restrict our attention to a family of special cases: we start with computing the monodromy of the fiber connected sum along two copies of the standard braid of index two, each of which is embedded in a copy of $D^{2} \times[0,1]$. Then we conclude by considering two copies of the standard braid of arbitrary index $n \in \mathbb{N}$.

For the remainder of Section $4 \mathrm{~B}$ let $D, D^{\prime}$ denote two copies of the unit disc $D^{2} \subset \mathbb{R}^{2}$ and let $D_{\varepsilon}(p)$ denote a disc of radius $\varepsilon>0$ centered at $p \in \mathbb{R}^{2}$. Let $\Sigma^{\prime}:=D \#^{2} D^{\prime}$ 

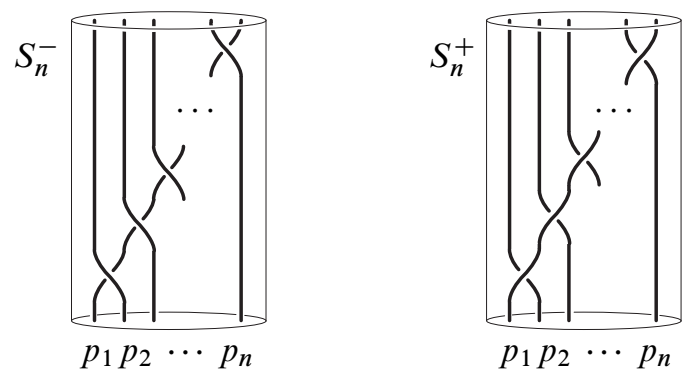

Figure 3: Description of the standard braids in $D^{2} \times[0,1]$. The framing is induced by the $x$-direction.

denote the 2-fold connected sum and recall that, for some sufficiently small $\varepsilon>0$, we may identify $\Sigma^{\prime}$ with

$$
\left(D \backslash \operatorname{int}\left(D_{\varepsilon}\left(-\frac{1}{2}, 0\right) \cup D_{\varepsilon}\left(\frac{1}{2}, 0\right)\right)\right) \cup\left(D^{\prime} \backslash \operatorname{int}\left(D_{\varepsilon}\left(-\frac{1}{2}, 0\right) \cup D_{\varepsilon}\left(\frac{1}{2}, 0\right)\right)\right) / \sim,
$$

where we identify $\partial D_{\varepsilon} \cup \partial D_{\varepsilon}$ in the first copy with its orientation reversed counterpart $-\left(\partial D_{\varepsilon} \cup \partial D_{\varepsilon}\right)$ in the latter. With the above identification in mind we define two framed knots in Figure 4:

$$
K, K^{\prime} \subset \Sigma^{\prime} \times S^{1}
$$

$\left(K^{\prime},+1\right)$
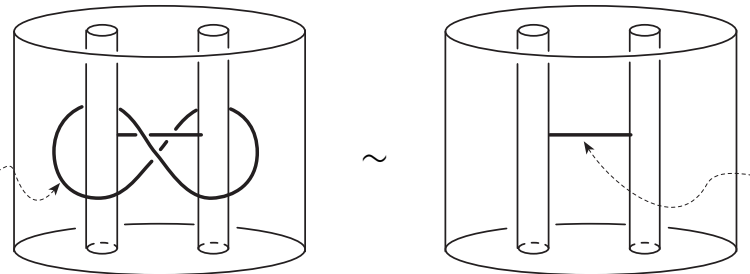

Figure 4: The knots $K$ and $K^{\prime}$ sitting in $\Sigma^{\prime} \times S^{1}$, where $\Sigma^{\prime}$ is understood as in Equation (4) (top and bottom are identified). Framings are measured with respect to an embedded copy of $\Sigma^{\prime}$.

Lemma 4.1 Denote by $\left(\Sigma^{\prime} \times S^{1}\right)\left(K, K^{\prime}\right)$ the result of surgery along $K, K^{\prime}$ (as defined above) with respect to their framings. Then the fiber connected sum along $S_{2}^{-} \subset D \times S^{1}$ and $S_{2}^{+} \subset D^{\prime} \times S^{1}$ (with respect to their natural framing and with respect to their parametrization described above) can be expressed as

$$
\left(D \times S^{1}\right) \#_{S_{2}^{-}, S_{2}^{+}}\left(D^{\prime} \times S^{1}\right) \cong\left(\Sigma^{\prime} \times S^{1}\right)\left(K, K^{\prime}\right) .
$$


Proof Consider the following explicit representation of $\Sigma^{\prime} \times S^{1}$ in $\mathbb{R}^{2} \times \mathbb{R} / \mathbb{Z}$ with coordinates $x, y, z$ : let $D$ and $D^{\prime}$ denote unit 2-discs in the $x y$-plane centered at the points $(0,2)$ and $(0,-2)$. Let $a_{1}, a_{2}, b_{1}, b_{2}, c \subset D \times S^{1}$ and $a_{1}^{\prime}, a_{2}^{\prime}, b_{1}^{\prime}, b_{2}^{\prime}, c^{\prime} \subset$ $D^{\prime} \times S^{1}$ denote the curves given in Figure 5 and let $N_{\boldsymbol{a}}, N_{\boldsymbol{a}^{\prime}}$ and $N_{\boldsymbol{b}}, N_{\boldsymbol{b}^{\prime}}$ denote $\varepsilon$ neighborhoods of $\boldsymbol{a}=a_{1} \cup a_{2}, \boldsymbol{a}^{\prime}=a_{1}^{\prime} \cup a_{2}^{\prime}$ and $\boldsymbol{b}=b_{1} \cup b_{2}, \boldsymbol{b}^{\prime}=b_{1}^{\prime} \cup b_{2}^{\prime}$ respectively. Then $\Sigma^{\prime} \times S^{1}$ can be described as

$$
\left(\left(D \times S^{1}\right) \backslash N_{\boldsymbol{a}}\right) \cup\left(\left(D^{\prime} \times S^{1}\right) \backslash N_{\boldsymbol{a}^{\prime}}\right) / \sim_{\partial N_{\boldsymbol{a}}},
$$

where we identify points $(x, y, z) \in \partial N_{\boldsymbol{a}}$ with their mirror image $(x,-y, z) \in \partial N_{\boldsymbol{a}^{\prime}}$. Note that the pair of $\operatorname{arcs} c, c^{\prime}$ descends to the closed curve $K$.
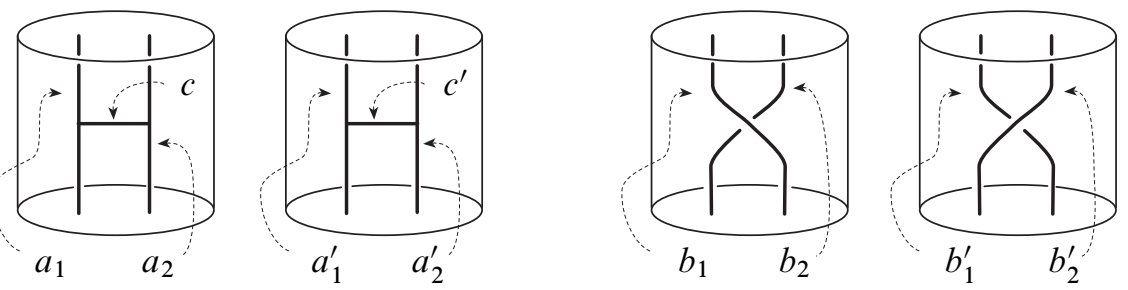

Figure 5: The $\operatorname{arcs} \boldsymbol{a}, \boldsymbol{a}^{\prime}, \boldsymbol{b}, \boldsymbol{b}^{\prime}, c, c^{\prime} \subset\left(D \times S^{1}\right) \sqcup\left(D^{\prime} \times S^{1}\right)$ (top and bottom are identified)

Denote by $H \subset M$ a neighborhood of the graph $\left(a_{1} \cup a_{2}\right) \cup c$. Choose $H^{\prime}$ to be the reflection of $H$ with respect to the $x z$-plane and note that $H^{\prime}$ provides a neighborhood of the graph $\left(a_{1}^{\prime} \cup a_{2}^{\prime}\right) \cup c^{\prime}$. Observe that the result $\left(\Sigma^{\prime} \times S^{1}\right)(K)$ of zero surgery along $K$ is

$$
\left(\left(D \times S^{1}\right) \backslash H\right) \cup\left(\left(D^{\prime} \times S^{1}\right) \backslash H^{\prime}\right) / \sim_{\partial H},
$$

where again we identify a point $(x, y, z) \in \partial H$ with its mirror $(x,-y, z) \in \partial H^{\prime}$.

Let us now perform the surgery along $K^{\prime}$. Isotope $K^{\prime}$ such that it lies on $\partial H$ sitting inside of $\left(D \times S^{1}\right) \backslash H$; see Equation (5) above. Note that the framing of $K^{\prime}$ and the framing induced by $\partial H$ agree. Let $\nu K^{\prime}=(-\varepsilon, \varepsilon) \times S^{1}$ denote a small open neighborhood of $K^{\prime}$ in $\partial H$. Remove a neighborhood $N_{K^{\prime}} \subset\left(\Sigma^{\prime} \times S^{1}\right)(K)$ of $K^{\prime}$ and observe that topologically the complement of $N_{K^{\prime}}$ is given by

$$
\left(\left(D \times S^{1}\right) \backslash H\right) \cup\left(\left(D^{\prime} \times S^{1}\right) \backslash H^{\prime}\right) / \sim_{\partial H \backslash \nu K^{\prime}},
$$

where we just identify points $(x, y, z) \in \partial H \backslash v K^{\prime}$ with their mirror $(x,-y, z) \in \partial H^{\prime}$. We will glue back the surgery torus $S^{1} \times D^{2}$ in two steps. Take $[0, \pi] \times D^{2} \subset S^{1} \times D^{2}$ (where we identify $S^{1} \equiv \mathbb{R} / 2 \pi \mathbb{Z}$ ) and attach it along $[0, \pi] \times \partial D^{2}$ to the closure of the neighborhood $\nu K^{\prime} \subset \partial H$, which we identify with $[-\varepsilon, \varepsilon] \times S^{1}$. Simultaneously attach 
$[\pi, 2 \pi] \times D^{2}$ along $[\pi, 2 \pi] \times \partial D^{2}$ to the mirror image of $\nu K^{\prime}$ on $\partial H^{\prime}$. We can actually picture this to be done inside of $H$ and $H^{\prime}$ respectively. Observe that the two pieces $\left([0, \pi] \times D^{2}\right),\left([\pi, 2 \pi] \times D^{2}\right)$, attached to the complement described in Equation (6) above, really descend to a solid torus. Moreover observe that we can understand the boundary of $N_{\boldsymbol{b}}$ as decomposes as $\left(\partial H \backslash v K^{\prime}\right) \cup\left(\{0, \pi\} \times D^{2}\right)$. Hence gluing back the surgery torus to the space given in Equation (6) gives

$$
\left(\left(D \times S^{1}\right) \backslash N_{\boldsymbol{b}}\right) \cup\left(\left(D^{\prime} \times S^{1}\right) \backslash N_{\boldsymbol{b}^{\prime}}\right) / \sim_{\partial N_{\boldsymbol{b}}},
$$

which describes $\left(D \times S^{1}\right) \#_{S_{2}^{+}}, S_{2}^{-}\left(D^{\prime} \times S^{1}\right)$. This completes the proof.
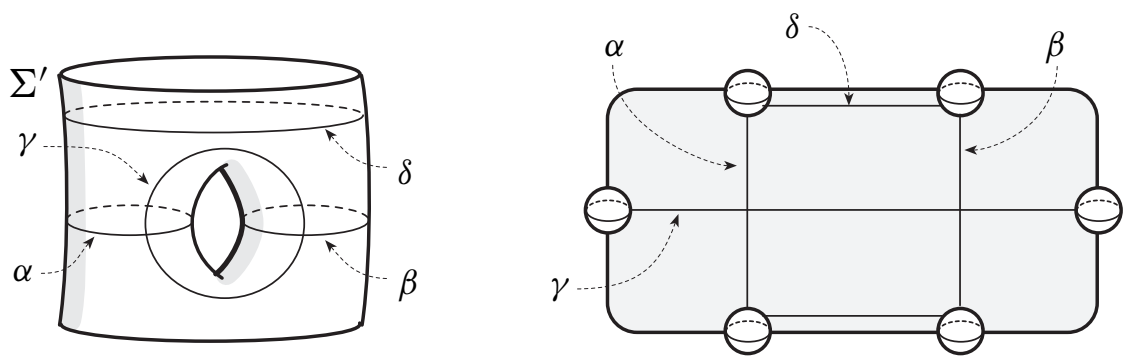

Figure 6: Two identifications of $\Sigma^{\prime}$ decorated with the curves $\alpha, \beta, \gamma, \delta$ used in Lemma 4.2 .

Recall that $\Sigma^{\prime}$ denotes the genus 1 surface with two boundary components understood as obtained by the 2 -fold connected sum of two copies $D, D^{\prime}$ of the 2-disc. Let $\alpha, \beta, \gamma, \delta \subset \Sigma^{\prime}$ be the curves described in Figure 6, where we identify $\Sigma^{\prime}$ such that cutting $\Sigma^{\prime}$ along $\alpha$ and $\beta$ decomposes as in Equation (4); the top half corresponds to $D$ and the bottom half to $D^{\prime}$. We would now like to express the surgery along $K, K^{\prime} \subset \Sigma^{\prime} \times S^{1}$ in the above description of $\left(D \times S^{1}\right) \#_{S_{2}^{-}}, S_{2}^{+}\left(D^{\prime} \times S^{1}\right)$ as a sequence of \pm 1 -surgeries along the above curves on $\Sigma^{\prime}$, where the framing is measured with respect to $\Sigma^{\prime}$.

Lemma 4.2 Let $\alpha, \beta, \gamma, \delta \subset \Sigma^{\prime}$ denote the curves described in Figure 6. Then setting $\psi=\left(\tau_{\alpha} \tau_{\beta} \tau_{\gamma}\right)^{2}\left(\tau_{\delta}\right)^{-1}$ we have

$$
\left(\Sigma^{\prime} \times S^{1}\right)\left(K, K^{\prime}\right) \cong \Sigma^{\prime}(\psi)
$$

where $\Sigma^{\prime}(\psi)$ denotes the mapping torus of $\psi$.

Proof Recall that in Lemma 4.1 we identified $\left(\Sigma^{\prime} \times S^{1}\right)\left(K, K^{\prime}\right)$ with the fiber connected sum $\left(D \times S^{1}\right) \#_{S_{2}^{+}}, S_{2}^{-}\left(D \times S^{1}\right)$. Observe that the latter space admits the structure of a $\Sigma^{\prime}$-fibration which is induced by the projection on $S^{1}$. The map $\psi$ is a 
factorization of the monodromy of this fibration into Dehn twists. A little caution is needed: in the following we compute the inverse of $\psi$, since in our computations we push arcs from the top to the bottom, not the other way around.

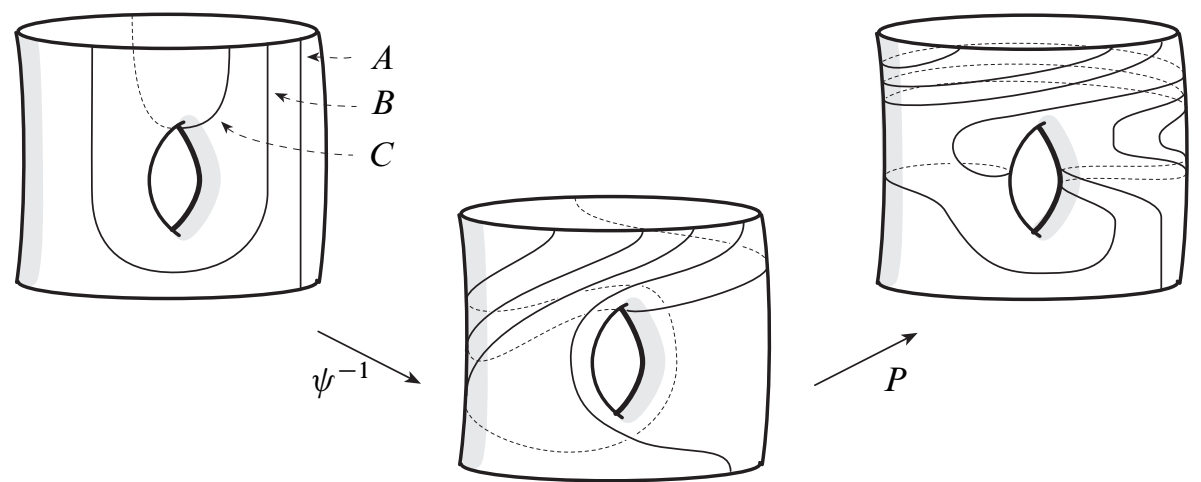

Figure 7: A cut system for $\Sigma^{\prime}$ and its image under $P \circ \psi^{-1}$.

Let $A, B, C \subset \Sigma^{\prime}$ denote the cut system given in the left part of Figure 7. The images of this cut system under $\psi^{-1}$ are given in the middle part of Figure 7. The images were computed as follows: note that we may understand $\Sigma^{\prime} \times[0,1]$ as obtained by thickening up the shaded area in Figure 6 (or Figure 8 respectively). A description of the knots $K, K^{\prime}$ with respect to this perspective is given in Figure 8. Understand the result of surgery $\left(\Sigma^{\prime} \times S^{1}\right)\left(K, K^{\prime}\right)$ on $K, K^{\prime}$ as embedded in the Kirby diagram given in Figure 8. We can now recover the cut system, chosen above, in the Kirby diagram and manipulate it using Kirby calculus. The actual computations are given in Figures 9 and 10. The shaded areas in the following figures indicate that an isotopy or a handle slide is performed.

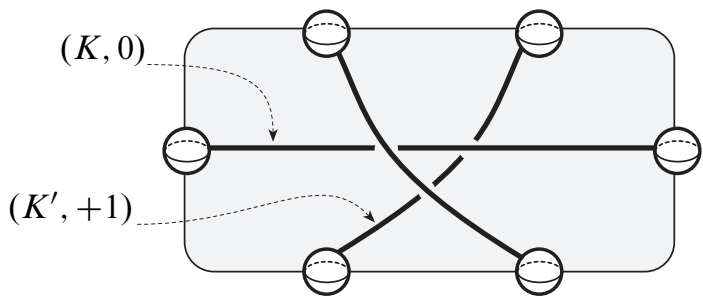

Figure 8: A Kirby diagram showing $K, K^{\prime}$ and $\Sigma^{\prime}$.

We could just compare these images with those under the inverse of $\left(\tau_{\alpha} \tau_{\beta} \tau_{\gamma}\right)^{2}\left(\tau_{\delta}\right)^{-1}$ and conclude that both agree up to isotopy, showing that $\psi=\left(\tau_{\alpha} \tau_{\beta} \tau_{\gamma}\right)^{2}\left(\tau_{\delta}\right)^{-1}$. However the usual way to compute the factorization of a monodromy map of a compact 

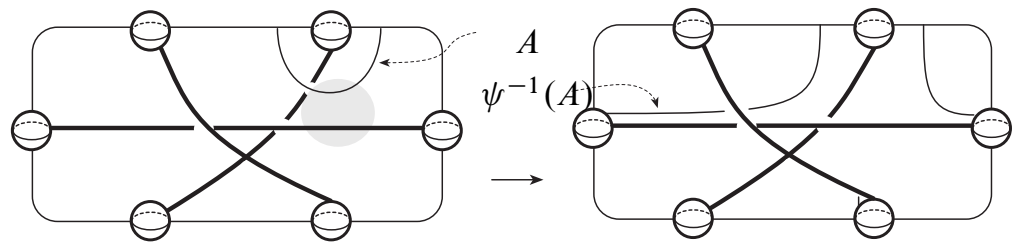

Figure 9: The image of $A$ under $\psi^{-1}$.
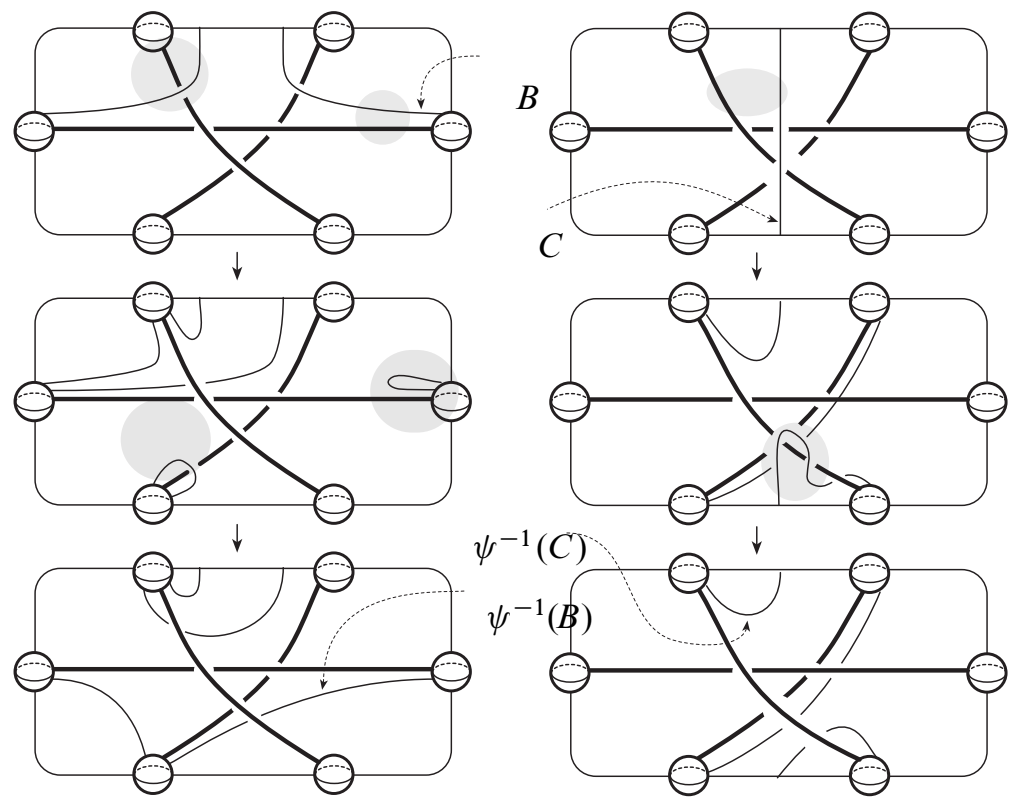

Figure 10: The images of $B$ and $C$ under $\psi^{-1}$.
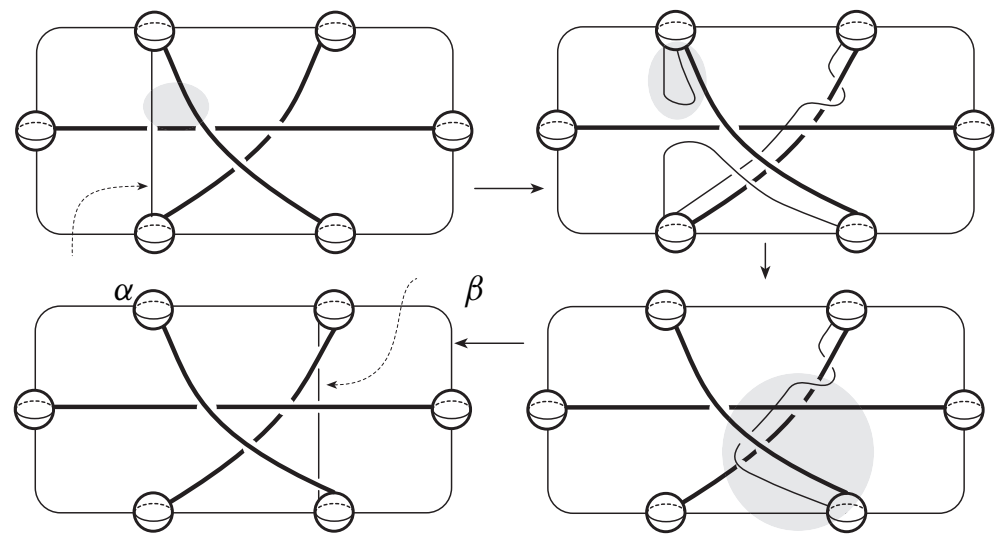

Figure 11: Computation of the image of $\alpha$ under $\psi^{-1}$. 
surface is by reducing it to the case of a self-diffeomorphism of a disc with punctures; see [7]. Referring to Figure 11 the image of $\alpha$ under $\psi^{-1}$ is given as $\beta$. Set

$$
P=\tau_{\gamma} \tau_{\beta} \tau_{\alpha} \tau_{\gamma}
$$

and note that $P$ maps $\beta$ to $\alpha$. Therefore $P \circ \psi^{-1}$ fixes the curve $\alpha$ and hence can now be interpreted as a self-diffeomorphism of the 3-fold punctured disc $D_{3}$ obtained by cutting $\Sigma^{\prime}$ along $\alpha$. Note that $A, B \subset \Sigma^{\prime}$ descends to a cut system of $D_{3}=\Sigma^{\prime} \backslash \alpha$. Therefore all the data of $P \circ \psi^{-1}$ is encoded in the images of $A, B \subset \Sigma^{\prime}$. The images of $A, B \subset \Sigma^{\prime}$ under $P \circ \psi^{-1}$ are given in the right part of Figure 7; see Figure 12 and Figure 13 for the actual computations. We conclude that

$$
P \circ \psi^{-1}=\tau_{\alpha}^{-1} \tau_{\beta}^{-1} \tau_{\delta}
$$

Therefore $\psi^{-1}$ is given by $\left(\tau_{\alpha}^{-1} \tau_{\beta}^{-1} \tau_{\delta}\right) \circ P^{-1}$, which, computing the inverse, is exactly what we intended to show.

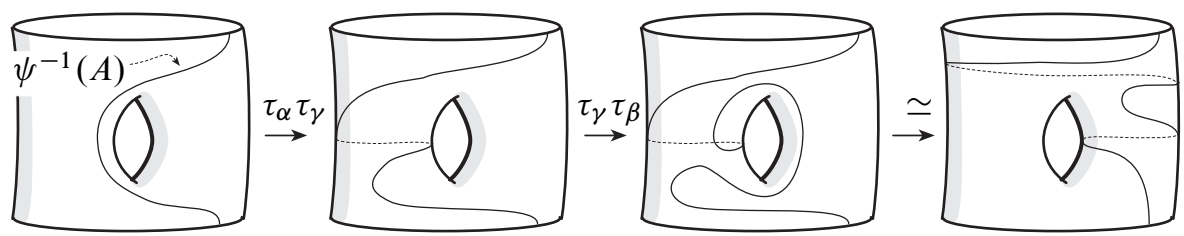

Figure 12: Computation of the image of $\psi^{-1}(A)$ under $P=\tau_{\gamma} \tau_{\beta} \tau_{\alpha} \tau_{\gamma}$.

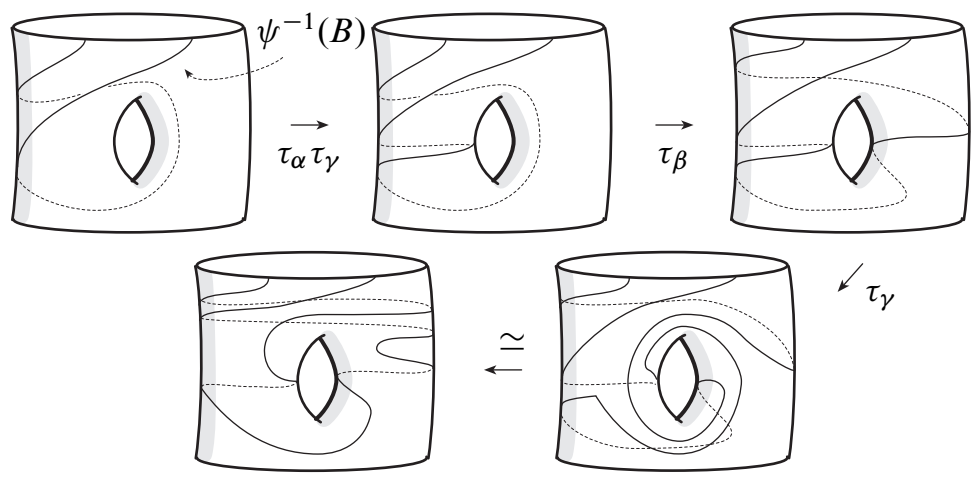

Figure 13: Computation of the image of $\psi^{-1}(B)$ under $P=\tau_{\gamma} \tau_{\beta} \tau_{\alpha} \tau_{\gamma}$.

With this in hand we are able to compute the monodromy for the case that $K_{0}$ and $K_{1}$ are chosen among the standard braids $S_{n}^{+}, S_{n}^{-}$given in Figure 3. Let $\Sigma:=D \#^{n} D^{\prime}$ denote the $n$-fold connected sum of two 2-discs $D$ and $D^{\prime}$. Let

$$
\alpha_{1}, \ldots, \alpha_{n}, \gamma_{1}, \ldots, \gamma_{n-1}, \delta_{1,2}^{(\prime)}, \ldots, \delta_{n-1, n}^{(\prime)} \subset \Sigma
$$


denote the curves indicated in Figure 14, where we identify $\Sigma$ such that cutting $\Sigma$ along $\alpha_{1} \cup \cdots \cup \alpha_{n}$ decomposes analogous to Equation (4); the top half corresponds to the first copy $D$ and the bottom half to the copy $D^{\prime}$.

Proposition 4.3 The fiber connected sum $\left(D \times S^{1} \#_{K_{0}, K_{1}} D^{\prime} \times S^{1}\right)$ along $K_{0} \subset D \times S^{1}$ and $K_{1} \subset D^{\prime} \times S^{1}$ can be expressed as the mapping torus $\Sigma(\phi)$, where the monodromy $\phi$ is given as follows:

(i) $\phi=\prod_{i=1}^{n-1} \tau_{\gamma_{i}} \tau_{\alpha_{i}} \tau_{\alpha_{i+1}} \tau_{\gamma_{i}}$ for the pair of knots $\left(K_{0}, K_{1}\right)=\left(S^{+}, S^{+}\right)$.

(ii) $\phi=\prod_{i=1}^{n-1}\left(\tau_{\gamma_{i}} \tau_{\alpha_{i}} \tau_{\alpha_{i+1}}\right)^{2}\left(\tau_{\delta_{i, i+1}}\right)^{-1}$ for the pair of knots $\left(K_{0}, K_{1}\right)=\left(S^{-}, S^{+}\right)$.

(iii) $\phi=\prod_{i=1}^{n-1} \tau_{\alpha_{i}} \tau_{\alpha_{i+1}}\left(\tau_{\gamma_{i}} \tau_{\alpha_{i}} \tau_{\alpha_{i+1}}\right)^{2}\left(\tau_{\delta_{i, i+1}}\right)^{-1}\left(\tau_{\delta_{i, i+1}^{\prime}}\right)^{-1}$ for the pair of knots $\left(K_{0}, K_{1}\right)=\left(S^{-}, S^{-}\right)$.

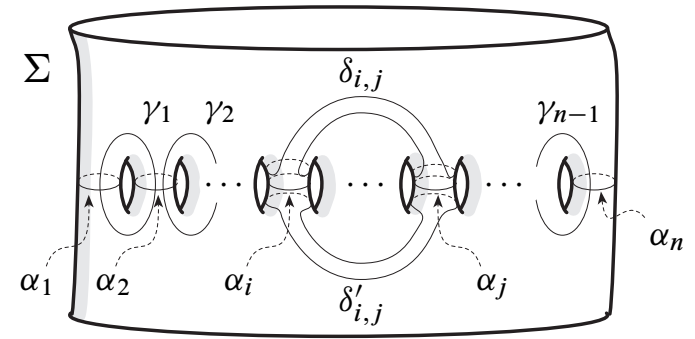

Figure 14: The curves used in Proposition 4.3 and Theorem 4.5.

Proof We start by proving the second part of the statement. Denote by $U_{k}$ the trivial braid of $k$-strands describing an $k$-component unlink. Now consider the following variation of the mapping torus defined by

$$
\Sigma\left(\psi_{1}, \ldots, \psi_{n-1}\right):=\left(\bigcup_{i=1}^{n} \Sigma \times[2 i-1,2 i]\right) / \sim,
$$

where we identify $(x, 2 i)$ with $\left(\psi_{i}(x), 2 i+1\right)$, for $i=1, \ldots, n-1$, and identify $(x, 2 n)$ with $(x, 1)$ for each $x \in \Sigma$. Note that $\Sigma($ id, $\ldots$, id) describes the $n$-fold fiber connected sum along $U_{n} \subset D \times S^{1}$ and $U_{n} \subset D^{\prime} \times S^{1}$. Now set

$$
\psi_{i}:=\left(\tau_{\gamma_{i}} \tau_{\alpha_{i}} \tau_{\alpha_{i+1}}\right)^{2}\left(\tau_{\delta_{i, i+1}}\right)^{-1} \text { for } i=1, \ldots, n-1 .
$$

The monodromy map $\psi_{i}, i=1, \ldots, n-1$, corresponds to the monodromy given in Lemma 4.2, where the curves $\alpha, \beta, \delta$ correspond to $\alpha_{i}, \alpha_{i+1}, \delta_{i, i+1}$. Hence it follows from Lemma 4.2 that $\Sigma\left(\psi_{1}\right.$, id,$\ldots$, id $)$ describes the $(n-1)$-fold fiber connected sum along $S_{2}^{-} \cup U_{n-2}$ and $S_{2}^{+} \cup U_{n-2}$. We can now consecutively turn $\Sigma\left(\psi_{1}\right.$, id, . , id $)$ 
into the desired fiber connected sum along $S_{n}^{-}$and $S_{n}^{+}$. With the same argument as above we conclude that $\Sigma\left(\psi_{1}, \ldots, \psi_{k}\right.$, id, $\ldots$, id $)$ describes the $(n-k)$-fold fiber connected sum along $S_{k}^{-} \cup U_{n-k}$ and $S_{k}^{+} \cup U_{n-k}$. Hence, the concatenation

$$
\phi_{S^{-}, S^{+}}:=\psi_{n-1} \circ \cdots \circ \psi_{1}
$$

describes the desired monodromy, which completes the proof of the second part.
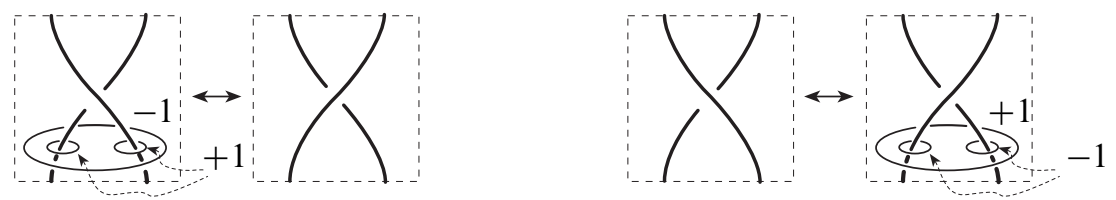

Figure 15: Switching between positive and negative crossings.

Recall that in Lemma 4.1 we consider the result of fiber summing the negative standard braid with the positive one. Observe that performing a surgery as indicated in the left part of Figure 15 (or right part of Figure 15 respectively) we can turn a negative (or positive respectively) crossing into a positive (or negative respectively) crossing; this is nothing but a certain Rolfsen twist. Imagine you want to add a positive twist using this surgery on the strands $i$ and $(i+1)$ sitting in $D \times S^{1}$, then the surgery reads

$$
R_{i}:=\tau_{\alpha_{i}}^{-1} \tau_{\alpha_{i+1}}^{-1} \tau_{\delta_{i, i+1}} \quad \text { for } i=1, \ldots, n-1 .
$$

Hence you could describe the fiber connected sum along $S_{n}^{+}$and $S_{n}^{+}$as $\Sigma\left(\psi_{1} \circ\right.$ $\left.R_{1}, \ldots, \psi_{n-1} \circ R_{-1}\right)$, ie

$$
\phi_{S^{+}, S^{+}}:=\left(\psi_{n-1} \circ R_{n-1}\right) \circ \cdots \circ\left(\psi_{1} \circ R_{1}\right) .
$$

This proves the first part of the theorem.

We follow the same strategy to prove the remaining part of the theorem. If you want to add a negative twist using this surgery on the strands $i$ and $(i+1)$ sitting in the other copy, namely $D^{\prime} \times S^{1}$, then the surgery reads

$$
R_{i}^{\prime}:=\tau_{\alpha_{i}} \tau_{\alpha_{i+1}} \tau_{\delta_{i, i+1}^{\prime}}^{-1} \text { for } i=1, \ldots, n-1 .
$$

Hence, you could describe the fiber connected sum along $S_{n}^{-}$and $S_{n}^{-}$as $\Sigma\left(\psi_{1} \circ\right.$ $\left.R_{1}^{\prime}, \ldots, \psi_{n-1} \circ R_{-1}^{\prime}\right)$, ie

$$
\phi_{S^{-}, S^{-}}:=\left(\psi_{n-1} \circ R_{n-1}^{\prime}\right) \circ \cdots \circ\left(\psi_{1} \circ R_{1}^{\prime}\right) .
$$

This shows the third part of the theorem and completes the proof. 


\section{C Final computation of the monodromy}

We almost have everything in place to compute the monodromy map of the fiber sum along multisections; see Equation (3). The last ingredient is the observation that we can put an $n$-fold section $K$ of a 3 -manifold $M$ with open book decomposition $(\Sigma, \phi)$ in a nicely adapted position as follows; note that this representation is not unique in general.

Lemma 4.4 Fix an embedded 2-disc $D \subset \Sigma$ in a sufficiently small neighborhood of $\partial \Sigma$. Let $K$ be an $n$-fold section of a 3-manifold $M$ with open book decomposition $(\Sigma, \phi)$. Then there is a diffeomorphism $\phi_{K}$ of $\Sigma$, and a diffeomorphism $M_{(\Sigma, \phi)} \rightarrow$ $M_{\left(\Sigma, \phi_{K} \circ \phi\right)}$ that maps $K$ to $S_{n}^{+}$.

Proof Express the complement of the binding $M \backslash B$ as the mapping torus $\Sigma \times$ $[0,1] / \sim_{\phi}$ and fix an embedded $2-$ disc $D^{2}$ in a sufficiently small neighborhood of $\partial \Sigma$. Observe that after an isotopy of $K$ we can assume that

- all intersection points $K \cap(\Sigma \times\{0\})=\left\{p_{1}, \ldots, p_{n}\right\}$ lie on the $x$-axis of $D^{2}$,

- $p_{1}<\cdots<p_{n}$,

- the permutation induced by $K$ is given by $\left(\begin{array}{llll}n & 1 & \cdots & (n-1))\end{array}\right)$.

After a second isotopy we can assume that for some small $\varepsilon>0$ the section $K$ corresponds to

- the standard braid $S_{n}^{+}$over $D^{2} \times[1-\varepsilon, 1] \subset \Sigma \times[1-\varepsilon, 1]$, and

- a pure braid $P$ over the remaining part $\Sigma \times[0,1-\varepsilon]$.

Note that the pure braid $P$ gives rise to an isotopy $\left(h_{t}\right)_{t \in[0,1]}$ of $\left\{p_{1}, \ldots, p_{n}\right\}$ within $\Sigma$. Moreover we can extend this isotopy of points to an isotopy $\left(H_{t}\right)_{t \in[0,1]}$ of $\Sigma$ for which we denote by $X_{t}$ the associated time dependent vector field on $\Sigma \times[0,1]$. Now consider the quotient space

$$
W:=((\Sigma \times[0,1]) \times[0,1]) / \sim_{H_{t} \circ \phi},
$$

where we identify $(p, t, 1) \sim\left(\left(H_{t} \circ \phi\right)(p), t, 0\right)$ for all $p \in \Sigma$ and $t \in[0,1]$. Note that this quotient is diffeomorphic to $\Sigma(\phi) \times[0,1]$. Furthermore consider the following vector field on $W$ defined by

$$
X:= \begin{cases}\partial_{t} & \text { for } s>1-\varepsilon, \\ (1-s /(1-\varepsilon)) \cdot X_{t}+\partial_{t} & \text { for } s \leq 1-\varepsilon .\end{cases}
$$

Observe that $X$ descends to a vector field on $W$. Furthermore the time 1 flow induces a diffeomorphism of $M$ that maps our original open book decomposition $(\Sigma, \phi)$ to $\left(\Sigma, H_{1} \circ \phi\right)$. In particular it maps $K$ to the $n$-fold section given by 
- the standard braid $S^{+}$in $D^{2} \times[1-\varepsilon, 1] \subset \Sigma \times[1-\varepsilon, 1]$, and

- the trivial pure braid $\left\{p_{1}, \ldots, p_{n}\right\} \times[0,1-\varepsilon]$ in $\Sigma \times[0,1-\varepsilon]$.

The map $H_{1}$ encodes all information about $K$ and we will denote it by $\phi_{K}$.

We now have everything in place to give an abstract description of the open book supporting the fiber sum along $K_{0}$ and $K_{1}$ described in Equation (3). For $i=0,1$ let $\left(\Sigma_{i}, \phi_{i}\right)$ denote an abstract description of the open book $\left(\pi_{i}, B_{i}\right)$ for which $K_{i}$ defines an $n$-fold section. Fix two embedded 2-discs $D \subset \Sigma_{0}$ and $D^{\prime} \subset \Sigma_{1}$ and denote by $\phi_{K_{0}}, \phi_{K_{1}}$ the maps associated to the knots $K_{0}$ and $K_{1}$ as described in Lemma 4.4. Moreover for $i=0,1$ there is a diffeomorphism $M_{\left(\Sigma_{i}, \phi_{i}\right)} \rightarrow M_{\left(\Sigma_{i}, \phi_{K_{i}} \circ \phi_{i}\right)}$ that maps $K_{i}$ to $S_{n}^{+}$. Hence we may endow $K_{0}$ and $K_{1}$ with the parametrization, as well as with the framing, induced by $S_{n}^{+}$. With respect to these choices we have

$$
M_{\left(\Sigma_{0}, \phi_{0}\right)} \#_{K_{0}, K_{1}} M_{\left(\Sigma_{1}, \phi_{1}\right)} \cong M_{\left(\Sigma_{0}, \phi_{K_{0}} \circ \phi_{0}\right)} \#_{S_{n}^{+}, S_{n}^{+}} M_{\left(\Sigma_{1}, \phi_{K_{1}} \circ \phi_{1}\right)} .
$$

Let $\Sigma_{0} \#^{n} \Sigma_{1}$ denote the $n$-fold connected sum of $\Sigma_{0}$ and $\Sigma_{1}$, where the connected sum operations are performed within $D, D^{\prime}$. With $\Sigma$ denoting the $n$-fold connected sum of the $2-\operatorname{discs} D, D^{\prime}$ we have a natural embedding

$$
\Sigma \subset\left(\Sigma_{0} \#^{n} \Sigma_{1}\right)
$$

Then combining Equation (7) and the first part of Proposition 4.3 we finally obtain the following description of the open book given in Equation (3).

Theorem 4.5 For $i=0,1$ let $\left(\Sigma_{i}, \phi_{i}\right)$ denote an abstract open book which admits an $n$-fold section $K_{i}$. Fix two embedded 2-discs $D \subset \Sigma_{0}$ and $D^{\prime} \subset \Sigma_{1}$ and denote by $\phi_{K_{0}}, \phi_{K_{1}}$ the maps associated to the knots $K_{0}$ and $K_{1}$ as described in Lemma 4.4. Moreover for $i=0,1$ there is a diffeomorphism $M_{\left(\Sigma_{i}, \phi_{i}\right)} \rightarrow M_{\left(\Sigma_{i}, \phi_{K_{i}} \circ \phi_{i}\right)}$ that maps $K_{i}$ to $S_{n}^{+}$. Hence we may endow $K_{0}$ and $K_{1}$ with the parametrization and framing induced by $S_{n}^{+}$. Moreover, the fiber connected sum $M_{\left(\Sigma_{0}, \phi_{0}\right)} \#_{K_{0}, K_{1}} M_{\left(\Sigma_{1}, \phi_{1}\right)}$ admits an open book description with page $\Sigma_{0} \#^{n} \Sigma_{1}$, the $n$-fold connected sum of $\Sigma_{0}$ and $\Sigma_{1}$, and monodromy given by

$$
\phi=\left(\prod_{i=1}^{n-1} \tau_{\gamma_{i}} \tau_{\alpha_{i}} \tau_{\alpha_{i+1}} \tau_{\gamma_{i}}\right) \circ\left(\phi_{K_{0}} \circ \phi_{0}\right) \circ\left(\phi_{K_{1}} \circ \phi_{1}\right),
$$

where $\alpha_{1}, \ldots, \alpha_{n}, \gamma_{1}, \ldots, \gamma_{n-1} \subset \Sigma$ denote the curves indicated in Figure 14 . 


\section{References}

[1] J W Alexander, A lemma on systems of knotted curves, Proc. Nat. Acad. Sci. USA 9 (1923) 93-95

[2] J B Etnyre, Lectures on open book decompositions and contact structures, from: "Floer homology, gauge theory, and low-dimensional topology", (D A Ellwood, P S Ozsváth, A I Stipsicz, Z Szabó, editors), Clay Math. Proc. 5, Amer. Math. Soc., Providence, RI (2006) 103-141 MR2249250

[3] H Geiges, An introduction to contact topology, Cambridge Studies in Advanced Mathematics 109, Cambridge Univ. Press (2008) MR2397738

[4] E Giroux, Géométrie de contact: de la dimension trois vers les dimensions supérieures, from: "Proc. ICM, Vol. II", (T Li, editor), Higher Ed. Press, Beijing (2002) 405-414 MR1957051

[5] Y Kanda, The classification of tight contact structures on the 3-torus, Comm. Anal. Geom. 5 (1997) 413-438 MR1487723

[6] B Ozbagci, M Pamuk, Full Lutz twist along the binding of an open book, Geom. Dedicata 147 (2010) 389-396 MR2660585

[7] V V Prasolov, A B Sossinsky, Knots, links, braids and 3-manifolds: an introduction to the new invariants in low-dimensional topology, Translations of Mathematical Monographs 154, Amer. Math. Soc., Providence, RI (1997) MR1414898

[8] W P Thurston, HE Winkelnkemper, On the existence of contact forms, Proc. Amer. Math. Soc. 52 (1975) 345-347 MR0375366

[9] J Van Horn-Morris, Constructions of open book decompositions, $\mathrm{PhD}$ thesis, University of Texas at Austin (2007) Available at http://math.stanford.edu/ jvanhorn/dissertation-jvhm.pdf

[10] C Wendl, A hierarchy of local symplectic filling obstructions for contact 3-manifolds, Duke Math. J. 162 (2013) 2197-2283 MR3102479

Mathematisches Institut, Universität zu Köln

Weyertal 86-90, D-50931 Köln, Germany

mirko.klukas@gmail.com

Received: 17 July 2012 Revised: 14 August 2015 
